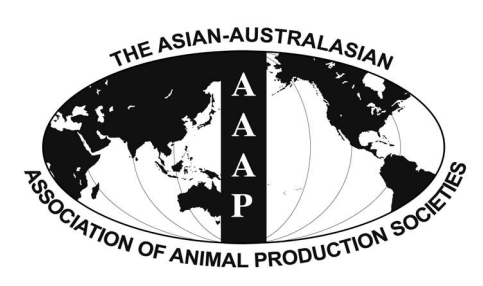

\title{
Rapamycin Influences the Efficiency of In vitro Fertilization and Development in the Mouse: A Role for Autophagic Activation
}

\author{
Geun-Kyung Lee ${ }^{1}$, Hyejin Shin ${ }^{1}$, and Hyunjung Jade Lim ${ }^{1,2, *}$ \\ ${ }^{1}$ Department of Biomedical Science and Technology, Institute of Biomedical Science and Technology, \\ Konkuk University, Seoul 143-701, Korea
}

\begin{abstract}
The mammalian target of rapamycin (mTOR) regulates cellular processes such as cell growth, metabolism, transcription, translation, and autophagy. Rapamycin is a selective inhibitor of mTOR, and induces autophagy in various systems. Autophagy contributes to clearance and recycling of macromolecules and organelles in response to stress. We previously reported that vitrified-warmed mouse oocytes show acute increases in autophagy during warming, and suggested that it is a natural response to cold stress. In this follow-up study, we examined whether the modulation of autophagy influences survival, fertilization, and developmental rates of vitrified-warmed mouse oocytes. We used rapamycin to enhance autophagy in metaphase II (MII) oocytes before and after vitrification. The oocytes were then subjected to in vitro fertilization (IVF). The fertilization and developmental rates of vitrifiedwarmed oocytes after rapamycin treatment were significantly lower than those for control groups. Modulation of autophagy with rapamycin treatment shows that rapamycin-induced autophagy exerts a negative influence on fertilization and development of vitrifiedwarmed oocytes. (Key Words: Vitrification, Mouse, Oocyte, Rapamycin, Development)
\end{abstract}

\section{INTRODUCTION}

Autophagy, a catabolic cellular process, contributes to recycling and clearance of macromolecules and organelles via the lysosomal degradation system (Boya et al., 2013). Damaged organelles or macromolecules are engulfed by autophagosomes that are enveloped by two layers of membranes, and these structures later fuse with lysosomes to form autolysosomes (Klionsky et al., 2012). Their contents are then degraded by various lysosomal enzymes, and the macromolecular building blocks and metabolites are recycled (Singh and Cuervo, 2011). Autophagy is ongoing at basal levels within cells, but is induced under unfavorable circumstances (Mizushima et al., 2008; Singh and Cuervo, 2011; Klionsky et al., 2012; Boya et al., 2013; Lim and Song, 2014). In mammalian reproduction and development, autophagy is implicated in the maintenance of primordial

\footnotetext{
* Corresponding Author: Hyunjung Jade Lim. Tel: +82-2-4504087, Fax: +82-2-2030-7809, E-mail: hlim@konkuk.ac.kr

${ }^{2}$ Department of Veterinary Medicine, Konkuk University, Seoul 143-701, Korea.

Submitted Sept. 11, 2015; Revised Oct. 1, 2015; Accepted Oct. 7, 2015
}

follicles, blastocyst cavitation, and fetal survival at birth (Lim and Song, 2014). Moreover, cold stress during vitrification and maternal diabetes induce autophagy in mammalian oocytes (Adastra et al., 2011; Bang et al., 2014; Tsukamoto et al., 2014). The activation of autophagy is important for turning over maternal proteins to newly synthesized embryonic ones during preimplantation embryonic development (Tsukamoto et al., 2008). Further, dormant blastocysts activate autophagy for their prolonged survival during delayed implantation (Lee et al., 2011a).

Cryopreservation of oocytes is a crucial tool for the preservation of fertility. Based on the conditions of freezing, there are two major methods for oocyte cryopreservation: slow freezing and vitrification (Saragusty and Arav, 2011). For vitrification, membrane-permeable cryoprotectants (CPAs) such as dimethyl sulfoxide (DMSO), ethylene glycol (EG), and propanediol (PROH) are combined with non-permeable agents such as sucrose and trehalose (Jo et al., 2011). Ultrastructures, nuclear changes, and spindle structures are common parameters used to assess the quality of cryopreserved oocytes (Gualtieri et al., 2011; MartínezBurgos et al., 2011). We recently showed that autophagy is

Copyright $@ 2016$ by Asian-Australasian Journal of Animal Sciences This is an open-access article distributed under the terms of the Creative Commons Attribution Non-Commercial License (http://creativecommons.org/licenses/by-nc/3.0/), which permits unrestricted non-commercial use, distribution, and reproduction in any medium, provided the original work is properly cited. 
induced in vitrified oocytes during warming, and that this induction is not associated with the presence of CPAs or sugars in the reagents used (Bang et al., 2014). This result showed, for the first time, that cold stress during freezing is a potent inducer of autophagy. However, it remained to be determined whether modulation of autophagy affects the subsequent fertilization and development favorably or adversely.

The mammalian target of rapamycin (mTOR) is a crucial regulator of cell growth and metabolism. mTOR is connected with diverse signaling pathways governing cell survival, growth, metabolism, transcription, translation, and autophagy. Therefore, mTOR is considered central to cellular signaling networks (Kapahi et al., 2010). Activated mTOR is phosphorylated, targeting downstream factors such as p70 S6 kinase (S6K) and eukaryotic initiation factor eIF4E-binding protein 1 for their subsequent phosphorylation and activation (Yu et al., 2010). mTOR is also a well-known negative regulator of autophagy. In fact, its inhibition with rapamycin, an immunosuppressive natural product, induces autophagy in various cell systems (Castedo et al., 2002; Yang and Klionsky, 2010). Phosphorylation of mTOR is mediated by S6K, and rapamycin suppresses mTOR-mediated translation by inhibiting the S6K activity (Chiang and Abraham, 2005). In metaphase II (MII) oocytes and preimplantation mouse embryos, autophagy and mTOR activation are inversely correlated, i.e., mTOR activity is high in MII oocytes and suppressed after fertilization, while autophagy displays the opposite trend (Tsukamoto et al., 2008; Yamamoto et al., 2014).

We previously showed that vitrified mouse oocytes show heightened autophagy during warming, and suggested that it is a natural adaptive response to cold stress (Bang et al., 2014). The use of 3-methyladenine, a widely used inhibitor of autophagy, during warming did not have a significant effect on the survival, fertilization, and development of vitrified-warmed oocytes (Bang et al., 2014). However, it remained to be identified whether enhanced or defective autophagy in MII oocytes affects the rates of in vitro fertilization (IVF) and development of vitrified oocytes. In this follow-up work, we investigate how the modulation of autophagy with rapamycin during vitrification affects the rates of vitrification-warming and IVF and development.

\section{MATERIALS AND METHODS}

\section{Mice}

All mice were maintained in strict accordance with the policies of the Konkuk University Institutional Animal Care and Use Committee (IACUC). This study was approved by the Konkuk University IACUC (approval number
KU14099). Mice were sacrificed under avertin to minimize suffering. Four-week-old virgin imprinting control region (ICR) female mice and ten-week-old ICR male mice were purchased from Orient-Bio (Seongnam, Korea).

\section{Collection of matured oocytes}

Four-to-five-week-old virgin ICR female mice were intraperitoneally injected with $7.5 \mathrm{IU}$ of pregnant mare's serum gonadotropin (Sigma-Aldrich, St. Louis, MO, USA) to induce folliculogenesis, followed by injection with 7.5 IU of human chorionic gonadotropin $48 \mathrm{~h}$ later to induce superovulation. Next, cumulus-oocyte complexes were collected from the oviducts 13 to $14 \mathrm{~h}$ post injection. The MII phase oocytes were retrieved in Quinn's Advantage Medium with HEPES (Sage, In Vitro Fertilization, Trumbull, CT, USA) containing $20 \%$ fetal bovine serum (FBS; Gibco, Grand Island, NY, USA) at $37^{\circ} \mathrm{C}$. The cumulus cells were then removed by using $300 \mu \mathrm{g} / \mathrm{mL}$ hyaluronidase (Sigma-Aldrich, USA).

\section{Vitrification and warming procedure}

The vitrification and warming procedure was essentially followed as described previously (Bang et al., 2014). The vitrification solution contains a mixture of EG (SigmaAldrich, USA) and DMSO (Sigma-Aldrich, USA). First, the oocytes were pre-equilibrated with a solution of $7.5 \% \mathrm{EG}$, $7.5 \% \mathrm{DMSO}$, and $20 \% \mathrm{FBS}$ for $2.5 \mathrm{~min}$; and then equilibrated with $15 \% \mathrm{EG}, 15 \%$ DMSO, $20 \% \mathrm{FBS}$, and 0.5 M sucrose (Fisher Scientific; Fair Lawn, NJ, USA) for $20 \mathrm{~s}$. Equilibrated oocytes were loaded onto cryotop (Kitazato Corporation, Shizuoka, Japan) and immersed in liquid nitrogen $\left(\mathrm{LN}_{2}\right)$. The vitrified oocytes were stored in $\mathrm{LN}_{2}$ for 2 weeks. After 2 weeks, they were warmed serially with sucrose solutions of decreasing concentrations $(0.5,0.25$, 0.125 , and $0 \mathrm{M}$ ) and $20 \%$ FBS for $2.5 \mathrm{~min}$ each. The vitrified-warmed oocytes were then cultured for 1 to $2 \mathrm{~h}$ in medium with HEPES containing 20\% FBS for recovery.

\section{Western blot analysis}

For each group, $100 \mathrm{MII}$ oocytes were directly collected in $35 \mu \mathrm{L} 2 \times$ sodium dodecyl sulfate (SDS) sampling buffer (100 mM Tris-Cl [pH 6.8], 4\% SDS, 20\% glycerol, $2 \% \beta-$ mercaptoethanol, and $0.2 \%$ bromophenol blue). The samples were boiled and centrifuged briefly, and then loaded onto $8 \%$ SDS-polyacrylamide gels. After electrophoresis, gels were blotted onto nitrocellulose membranes (Millipore, Billerica, MA, USA). These membranes were then blocked with 5\% skimmed milk in Tris-base saline for $1 \mathrm{~h}$ at room temperature and incubated with primary antibodies at $4^{\circ} \mathrm{C}$ overnight. They were then incubated with peroxidase-conjugated secondary antibodies (GenDEPOT, Barker, TX, USA) diluted to 1:10,000 for $1 \mathrm{~h}$. Chemiluminescence signals were detected using the 
LAS3000 system (Fujifilm, Tokyo, Japan) and quantified by Multi Gauge software (Fuji Film, Japan). The primary antibodies used were: rabbit polyclonal anti-total-mTOR (1:1,000, Cell Signaling Technology, Danvers, MA, USA), rabbit polyclonal anti-p-mTOR (Ser 2448) (1:1,000, Cell Signaling Technology, USA), and mouse monoclonal anti$\alpha$-tubulin (1:1,000, Sigma-Aldrich, USA).

\section{Immunofluorescence staining and confocal microscopy}

Cultured oocytes were fixed and permeabilized with 4\% paraformaldehyde with $0.1 \%$ Triton $\mathrm{X}-100$ in phosphatebuffered saline (PBS) for $20 \mathrm{~min}$, and washed twice with $0.1 \%$ Triton $\mathrm{X}-100$ in PBS for $5 \mathrm{~min}$ each. The oocytes were then blocked in $2 \%$ bovine serum albumin (BSA)/PBS drop for $1 \mathrm{~h}$. Next, they were incubated with the primary antibody drop containing either anti-p-mTOR (rabbit polyclonal, 1:200; Cell Signaling Technology, USA), antiLight chain 3 beta (LC3b) (rabbit polyclonal, 1:400; Cell signaling Technology, USA) or anti-Beclin1 (rabbit polyclonal, 1:200; Novus Biologicals, Littleton, CO, USA) in $2 \% \mathrm{BSA} / \mathrm{PBS}$ overnight at $4^{\circ} \mathrm{C}$. The oocytes were washed thrice in $2 \% \mathrm{BSA} / \mathrm{PBS}$ for $5 \mathrm{~min}$ each, and incubated with Alexa Fluor 488 chicken anti-rabbit secondary antibody (1:250; Molecular Probes, Trumbull, CT, USA) in $2 \% \mathrm{BSA} / \mathrm{PBS}$ for $40 \mathrm{~min}$. After three washes, the oocytes were counterstained with TO-PRO-3-iodide (1:250; Life Technologies, Invitrogen, Carlsbad, CA, USA). They were then washed in $2 \%$ BSA/PBS, directly placed on a glass slide, and covered with a glass coverslip that was sealed with transparent nail polish. Rabbit IgG was used as mock control. Immunofluorescence images were obtained using LSM710 confocal microscope (Carl Zeiss, Oberkochen, Germany), and analyzed using Zen 2009 Light Edition, a platform associated with the confocal microscope. For quantification of p-mTOR puncta, each oocyte was imaged at four planes ( $3 \mu \mathrm{m}$ intervals) using LSM710 confocal microscope (Carl Zeiss, Germany).

\section{Rapamycin treatment of oocytes and experimental}

\section{groups}

Rapamycin (Millipore) was dissolved in DMSO (Sigma-Aldrich, USA). We set up two experimental schemes as shown in Figure 1. Experiment 1: rapamycinpretreated oocytes were vitrified and warmed, and recovered by culturing for 1 to $2 \mathrm{~h}$ at $37^{\circ} \mathrm{C}$ in $5 \% \mathrm{CO}_{2}$. They were then fertilized in human tubal fluid (HTF) containing $10 \%$ substitute protein serum (SPS) for $6 \mathrm{~h}$ in vitro, washed, and cultured in KSOM-AA (Millipore, Danvers, MA, USA) at $37^{\circ} \mathrm{C}$ in $5 \% \mathrm{CO}_{2}$. Experiment 2: the vitrified oocytes were warmed in warming solutions containing $100 \mathrm{nM}$ rapamycin, and recovered by culturing in medium with HEPES containing 20\% FBS and $100 \mathrm{nM}$ rapamycin for 1 to $2 \mathrm{~h}$ at $37^{\circ} \mathrm{C}$ in $5 \% \mathrm{CO}_{2}$. Control oocytes underwent the same procedure with solutions containing the same volume of DMSO. Vitrified-warmed oocytes were fertilized in vitro, washed, and cultured in $\mathrm{KSOM}-\mathrm{AA}$ at $37^{\circ} \mathrm{C}$ in $5 \% \mathrm{CO}_{2}$.

\section{In vitro fertilization and embryo culture}

IVF was performed according to the procedure described by Cha et al. (2011). Epididymal sperms were obtained from 10 to 11 week-old male ICR mice. For capacitation, a drop of sperm suspension was added in Quinn's Advantage Fertilization Medium (HTF, Sage, Trumbull, CT, USA) containing 10\% SPS (Sage) for 90 $\min$ at $37^{\circ} \mathrm{C}$ in $5 \% \mathrm{CO}_{2}$. For IVF, the capacitated sperm suspension was added to oocytes in HTF containing $10 \%$ SPS. After $6 \mathrm{~h}$, the oocytes were washed four times with HTF containing $10 \%$ SPS and finally, with KSOM-AA medium. They were then cultured in KSOM-AA drops overnight. Fertilization was confirmed $24 \mathrm{~h}$ later by observing two-cell embryos. They were cultured for 5 days to assess embryonic development.

\section{Statistical analyses}

All data were graphed using GraphPad Prism (version 5). The statistical analyses were performed using Student's $t$-test (one-tailed). The criteria for statistical significance
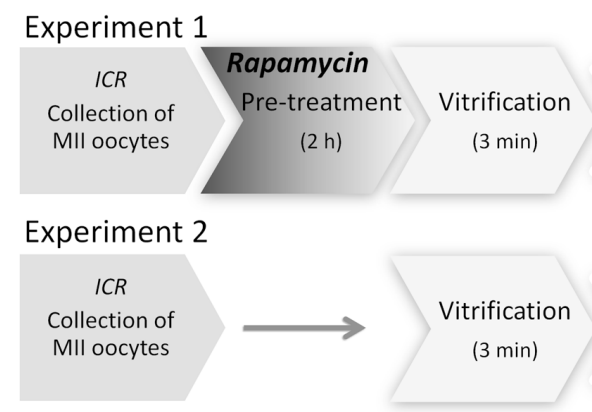
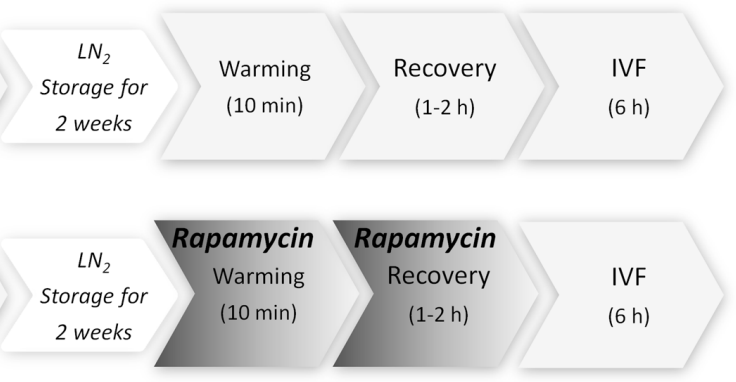

Figure 1. The experimental schemes. Metaphase II (MII) oocytes were collected from ICR mice and used in two independent experiments (Experiment 1 and 2). Experiment 1: MII oocytes were treated with rapamycin (100 or $250 \mathrm{nM})$ prior to vitrification. They were then vitrified for 2 weeks, warmed, and subjected to in vitro fertilization (IVF). Experiment 2: Vitrified MII oocytes were warmed and recovered in the presence of rapamycin $(100 \mathrm{nM})$ for 80 to $90 \mathrm{~min}$ and subjected to IVF. 
were: $* \mathrm{p}<0.05, * * \mathrm{p}<0.01, * * * \mathrm{p}<0.001$.

\section{RESULTS}

\section{Timing of rapamycin treatment: experimental groups}

Rapamycin indirectly induces autophagy by inhibiting mTOR, a major suppressor of autophagy (Neufeld, 2010). To identify whether induction of autophagy by rapamycin treatment has a positive or negative effect on the survival, fertilization, and development of vitrified-warmed mouse oocytes, rapamycin was added at two different points as follows: Experiment 1, prior to vitrification; and Experiment 2, during warming (Figure 1). The effects of rapamycin on mTOR phosphorylation and autophagic induction were confirmed by western blotting and immunofluorescence staining (see sections below).

\section{mTOR in fresh and vitrified-warmed metaphase II} oocytes

Prior to assessing the effect of rapamycin on IVF and development of vitrified oocytes, we first examined whether our treatment regimen was effective enough to suppress mTOR phosphorylation in MII oocytes. To address this, we performed immunofluorescence staining and western blotting of total and phospho(p)-mTOR in MII oocytes treated with $100 \mathrm{nM}$ rapamycin. As shown in Figure 2, pmTOR signals are observed as puncta in the ooplasm (Sancak et al., 2008). The overall expression of p-mTOR in rapamycin-treated oocytes were lower than that in control (fresh and DMSO-treated). Figure 2B illustrates western blotting of p-mTOR; p-mTOR levels decreased in both MII oocytes treated with $100 \mathrm{nM}$ rapamycin for $2 \mathrm{~h}$ (Experiment 1), and vitrified-warmed ones treated with the same during warming (Experiment 2). As shown, rapamycin Rapamycin treatment reduces phosphorylation of significantly reduced the number of p-mTOR puncta
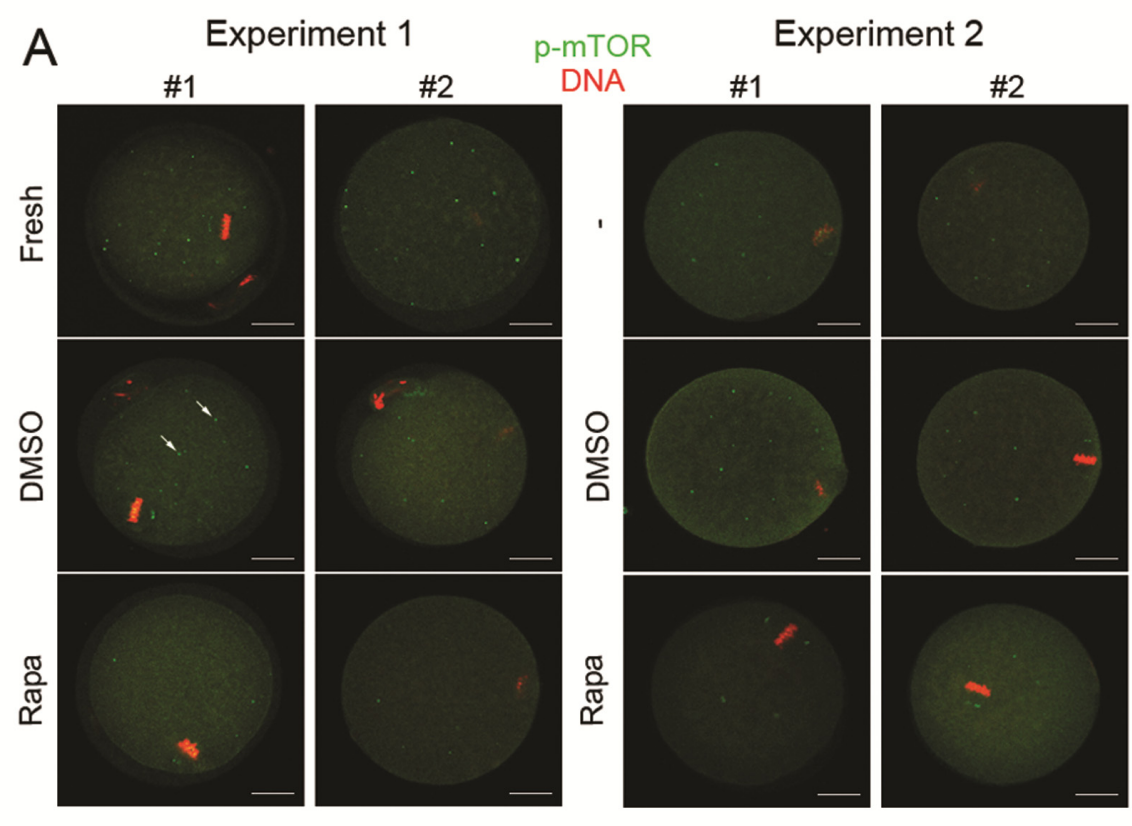

B
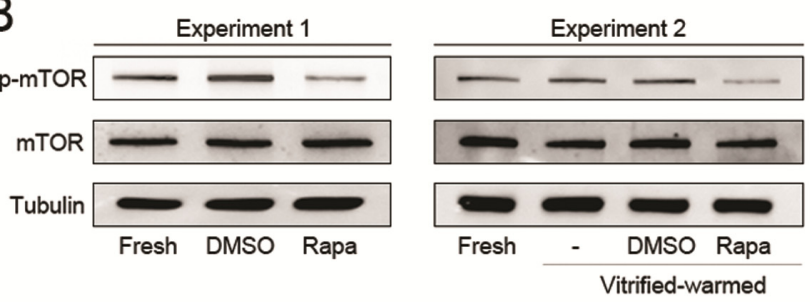

Figure 2. Rapamycin treatment suppresses phosphorylation of mammalian target of rapamycin (mTOR) in metaphase II (MII) oocytes. (A) Immunofluorescence staining of p-mTOR in oocytes. Rapamycin was used at $100 \mathrm{nM}$. Control oocytes were treated with the same amount of vehicle (dimethyl sulfoxide). Oocytes were stained with rabbit polyclonal anti-p-mTOR antibodies (1:200). Alexa Flour 488 chicken anti-rabbit IgG was used as the secondary antibody. DNA was stained with TO-PRO-3-iodide (1:250). Green, p-mTOR; red, DNA. Scale bar, $20 \mu \mathrm{m}$. The experiment was repeated 6 times using at least 20 oocytes in each group. Two representative images from each group are shown. Rabbit IgG was used as mock control and did not produce any signal (data not shown). While arrows indicate some examples of p-mTOR puncta. (B) Western blotting of mTOR in oocytes. MII oocytes (100 per group) in Experiments 1 and 2 were collected in SDS sampling buffer. Total lysates were loaded onto $8 \%$ polyacrylamide gels and subjected to western blotting with antimTOR, anti-phospho (p)-mTOR, and anti- $\alpha$-tubulin antibodies. This experiment was repeated thrice with similar results. 
formed in both groups compared with that in the control groups. Collectively, these results confirm that the treatment condition adopted effectively reduces phosphorylation of mTOR in fresh and vitrified-warmed oocytes.

\section{Rapamycin treatment in metaphase II oocytes prior to} vitrification reduces fertilization rate

We then confirmed if rapamycin treatment induces autophagic activation in the MII oocytes. In Experiment 1, MII oocytes were incubated with 100 or $250 \mathrm{nM}$ rapamycin in DMSO for $2 \mathrm{~h}$ prior to vitrification. The oocytes were then subjected to immunofluorescence staining with antiLC3 antibody. LC3 is a marker for autophagic activation and generally exhibits puncta formation in the cytoplasm (Mizushima et al., 2004). As shown in Figure 3A, treatment with rapamycin increased the LC3 immunofluorescence at both 100 and $250 \mathrm{nM}$ concentrations, the difference being more evident in case of the former. The oocytes with or without rapamycin treatment were then subjected to vitrification ( 2 weeks), warming, and IVF. While the survival rates of vitrified-warmed oocytes were similar among the groups (Figure 3B), fertilization rates were markedly reduced (Figure 4). Developmental rate to the blastocyst stage was also reduced in the $250 \mathrm{nM}$ rapamycintreated group. Thus, exposure to rapamycin prior to vitrification at MII increases autophagy, and negatively affects fertilization and developmental rates in vitro.

\section{Rapamycin treatment in vitrified metaphase II oocytes} during warming mildly affects fertilization and developmental rates

MII oocytes in Experiment 2 received rapamycin treatment $(100 \mathrm{nM})$ during warming and a recovery time of 80 to $90 \mathrm{~min}$ after vitrification (Figure 1). To confirm enhanced autophagic response in the rapamycin-treated oocytes, immunofluorescence staining of LC3 and Beclin1 was performed (Figures 5A and B). As shown, expressions of both the markers increased in presence of rapamycin. These oocytes were then subjected to IVF and development. As Figure 6 illustrates, fertilization and developmental rates significantly decreased in the rapamycin-treated group. Thus, rapamycin-caused enhanced autophagy beyond
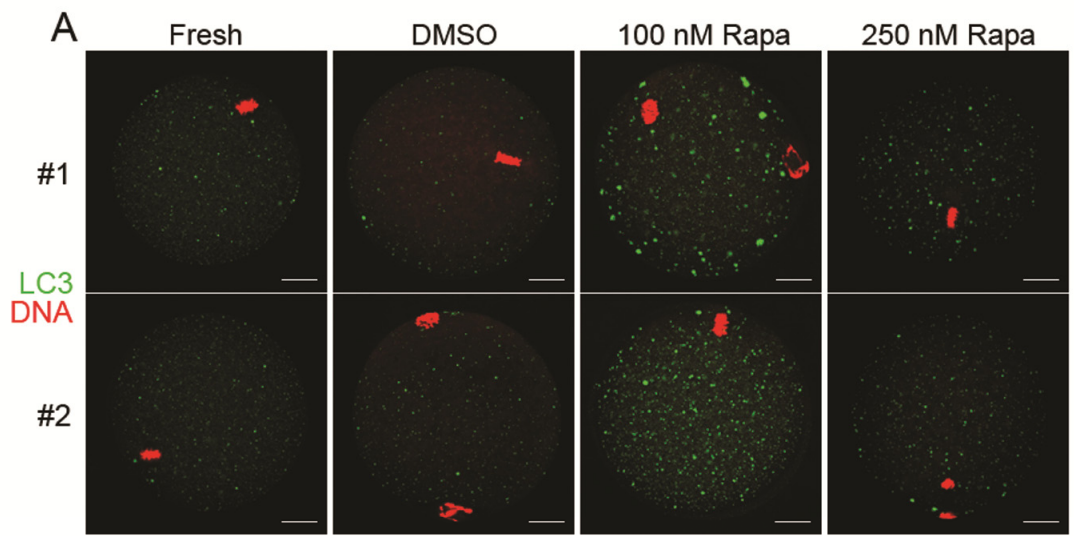

Rabbit IgG
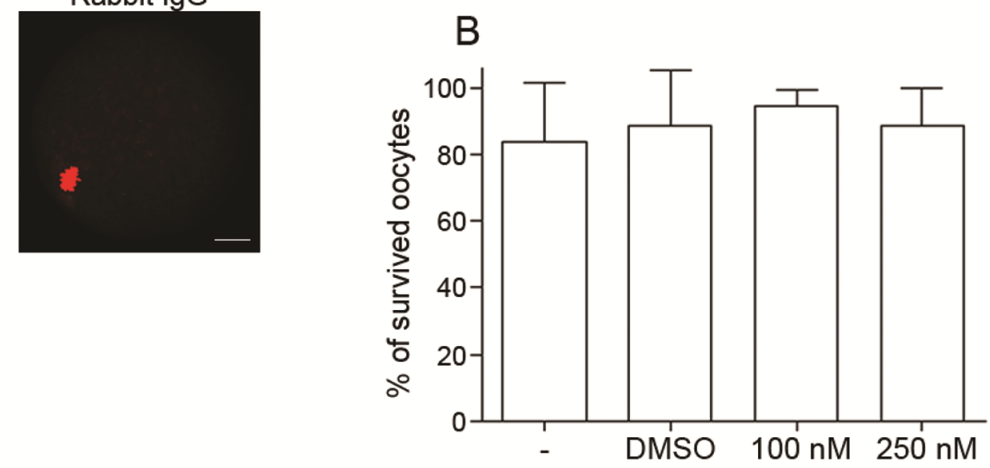

Figure 3. Experiment 1: Effect of rapamycin treatment prior to vitrification. (A) Immunofluorescence staining of light chain 3 (LC3). Rapamycin was used at 100 or $250 \mathrm{nM}$. Control oocytes were treated with the same amount of dimethyl sulfoxide (vehicle). After rapamycin pretreatment for $2 \mathrm{~h}$, metaphase II (MII) oocytes were washed with medium with HEPES containing $20 \%$ fetal bovine serum and stained with rabbit polyclonal anti-LC3b antibodies (1:400). Alexa Flour 488 chicken anti-rabbit IgG was used as secondary antibody. DNA was stained with TO-PRO-3-iodide $(1: 250)$ and rabbit IgG was used as mock control. Green, LC3; red, DNA. Scale bar, $20 \mu \mathrm{m}$. The experiment was repeated 5 times using at least 20 oocytes in each group. Two representative images from each group are shown. (B) Effect of rapamycin pretreatment on survival of vitrified-warmed oocytes. The number of oocytes in each group is shown in the table of Figure 4. 

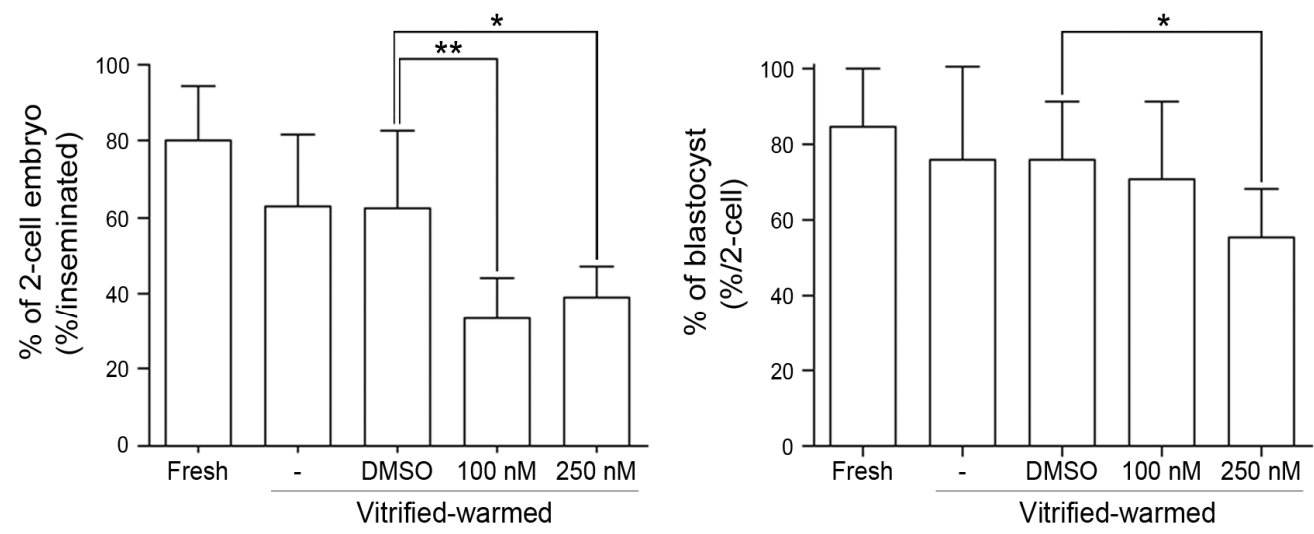

\begin{tabular}{ccccccc}
\hline \multirow{2}{*}{ Group } & \multicolumn{5}{c}{ Number of } \\
\cline { 2 - 7 } & $\begin{array}{c}\text { Vitrified } \\
\text { oocytes }\end{array}$ & $\begin{array}{c}\text { Survived } \\
\text { oocytes (\%) }\end{array}$ & $\begin{array}{c}\text { Inseminated } \\
\text { oocytes }\end{array}$ & $\begin{array}{c}\text { Two-cell embryo } \\
\text { (\%/inseminated) }\end{array}$ & $\begin{array}{c}\text { Blastocysts } \\
\text { (\%/two-cell) }\end{array}$ \\
\hline \multirow{2}{*}{ Fresh } & - & - & 226 & $187(82.7)$ & $156(83.4)$ \\
\hline \multirow{2}{*}{$\begin{array}{c}\text { Vitrified } \\
\text {-warmed }\end{array}$} & DMSO & 218 & $194(89.0)$ & 194 & $133(68.6)$ & $96(72.2)$ \\
\cline { 2 - 7 } & 100 nM Rapa & 223 & $214(96.0)$ & 214 & $74(34.6)$ & $49(66.2)$ \\
\cline { 2 - 7 } & 250 nM Rapa & 224 & $204(91.1)$ & 204 & $81(39.7)$ & $44(54.3)$ \\
\hline
\end{tabular}

Figure 4. Effect of rapamycin pretreatment on fertilization and development of vitrified-warmed oocytes. Graphs show the percentage of 2-cell embryos and blastocysts after in vitro fertilization. The table shows the total number and percentage of oocytes that were used in the barograms. Data represent the mean of six independent experiments $(n=6)$, while error bars represent the mean \pm standard deviation (SD). Statistical significance was analyzed by Student's $t$ test (one-tailed). ${ }^{*} \mathrm{p}<0.05,{ }^{* *} \mathrm{p}<0.01$.

physiological levels during warming after vitrification exerts a negative effect on fertilization and development.

\section{DISCUSSION}

In this work, we report that rapamycin treatment causes enhancement of autophagy beyond the physiological level in MII oocytes and that this in turn negatively influences both fertilization and developmental rates of vitrifiedwarmed mouse oocytes. Rapamycin is a natural product of the bacterium Streptomyces hygroscopicus with immunosuppressive effects (Heitman et al., 1991). As a suppressor of mTOR complex 1 (mTORC1), rapamycin is widely used to induce autophagy (Choi et al., 2014) in oocytes and embryos (Lee et al., 2011b; Song et al., 2014). Meanwhile, mTOR also has several indicated roles in oocyte biology (Lee et al., 2012; Kogasaka et al., 2013; Mayer et al., 2014). A recent work showed that inhibition of mTOR activity during meiotic progression of immature oocytes causes spindle anomaly and chromosome misalignment (Susor et al., 2015). Rapamycin, used at 100 $\mathrm{nM}$ for $12 \mathrm{~h}$, showed an inhibitory effect on the translation of specific transcripts associated with meiotic progression, but did not interfere with the overall protein synthesis (Susor et al., 2015). In our study, we used rapamycin in mature MII oocytes for 1 to $2 \mathrm{~h}$ before and after vitrification, and observed that fertilization and developmental rates are affected by this brief exposure (Figures 4 and 6). Such short exposure was sufficient to reduce mTOR phosphorylation and increase autophagic activation, and did not cause distinct morphological or cellular abnormalities (Figures 2 to 5) as in immature mouse oocytes (Susor et al., 2015). Our observations, however, do not preclude the possibility that observed effects of rapamycin may have caused undetected side effects.

MII oocytes have been shown to maintain low levels of autophagy until fertilization (Tsukamoto et al., 2008). Within $4 \mathrm{~h}$ of fertilization, autophagy is activated to produce new maternal proteins needed for embryonic development. Therefore, defective autophagy in oocytes lacking Atg5 gene negatively affects preimplantation embryonic development by impeding protein turnover during oocyte-to-embryo transition (Tsukamoto et al., 2008). A recent work by Yamamoto et al. (2014) has shown that the mTOR complex 1 (mTORC1) activity is relatively high in MII oocytes and decreases rapidly after fertilization, exhibiting an inverse correlation with autophagy (Yamamoto et al., 2014). The authors used alternative inhibitors of mTOR such as Torin1 and PP242 to increase autophagy in MII oocytes and embryos, but were unsuccessful. Thus, they suggested that fertilizationinduced autophagy is independent of mTORC1 activity 
A

Vitrified-warmed oocytes
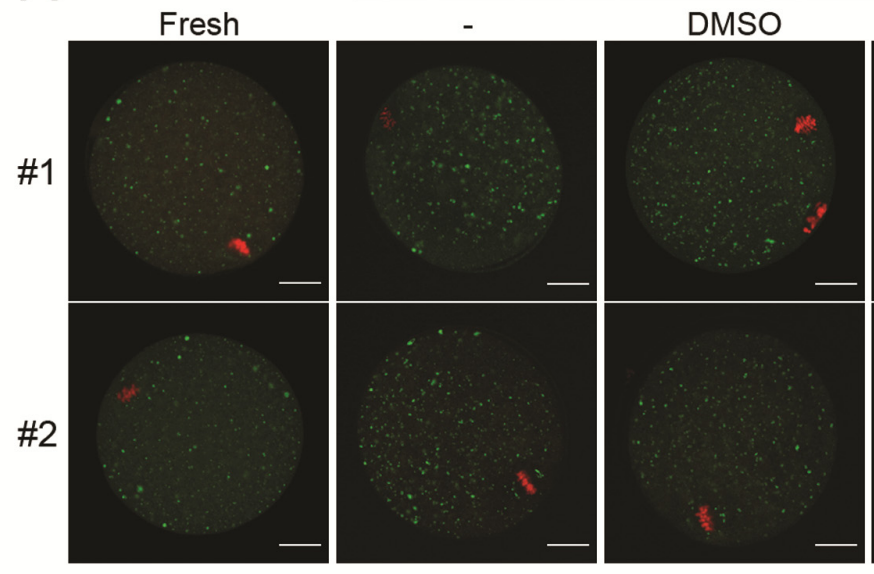

100 nM Rapa

B
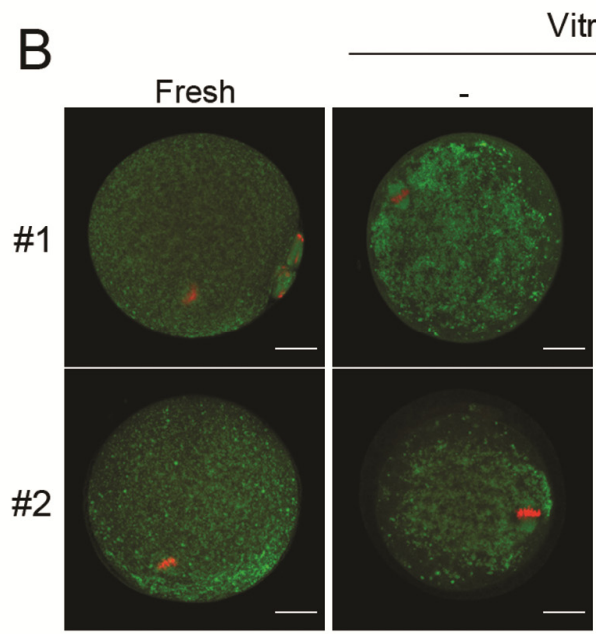

Vitrified-warmed oocytes

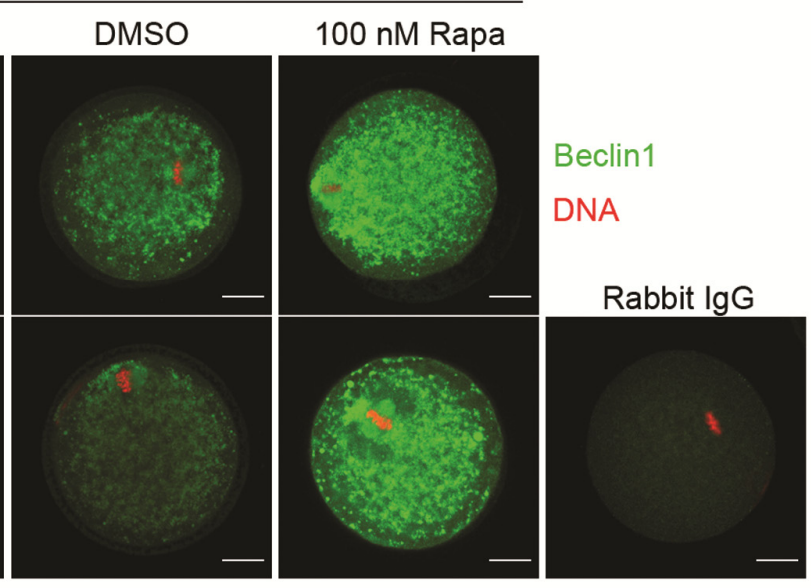

Figure 5. Experiment 2: Effect of rapamycin treatment during warming and recovery of vitrified oocytes. (A) Immunofluorescence staining of light chain 3 (LC3) in oocytes treated with rapamycin during warming and recovery after vitrification. Rapamycin was used at $100 \mathrm{nM}$. Control oocytes were treated with the same amount of dimethyl sulfoxide (vehicle). Oocytes were stained with rabbit polyclonal anti-LC3b antibodies (1:400). Rabbit IgG was used as mock control. Green, LC3; red, DNA. Scale bar, $20 \mu$ m. The experiment was repeated 3 times using at least 20 oocytes in each group. Two representative images from each group are shown. (B) Immunofluorescence staining of Beclin1 in oocytes treated with rapamycin during warming and recovery after vitrification. Oocytes were stained with rabbit polyclonal anti-Beclin1 antibodies (1:200). Alexa Flour 488 chicken anti-rabbit IgG was used as secondary antibody. DNA was stained with TO-PRO-3-iodide (1:250). The experiment was repeated 3 times using at least 15 oocytes in each group. Two representative images from each group are shown.

(Yamamoto et al., 2014). In our experiment, rapamycin treatment increased autophagy in MII oocytes, but this induction is not essentially associated with fertilization. Thus, the distinctive results are most likely caused by differences in timing of treatment regimen as well as the type and dose of inhibitors used. Rapamycin was also used in a study on porcine oocytes, which induced slight increases in LC3-II expression. This treatment was shown to improve cytoplasmic and nuclear maturation in porcine oocytes by increasing autophagic activation (Song et al., 2014). Thus, mTOR is associated with regulation of autophagy in mammalian oocytes.

In both our experiments (Experiments 1 and 2), we observed that a short exposure of MII oocytes to rapamycin prior to fertilization increases autophagy and this, in turn, is associated with decreased fertilization and developmental rates (Figures 4 and 6). The fertilization rate was more affected by this treatment, suggesting that premature increase in autophagy at fertilization is damaging. As mentioned before, the warming process after vitrification promotes autophagic activation (Bang et al., 2014). Thus, as we assumed in our previous work (Bang et al., 2014), autophagic induction during warming is likely a natural response to cold stress.

In conclusion, our data suggest that heightened autophagy beyond a physiological response during warming 

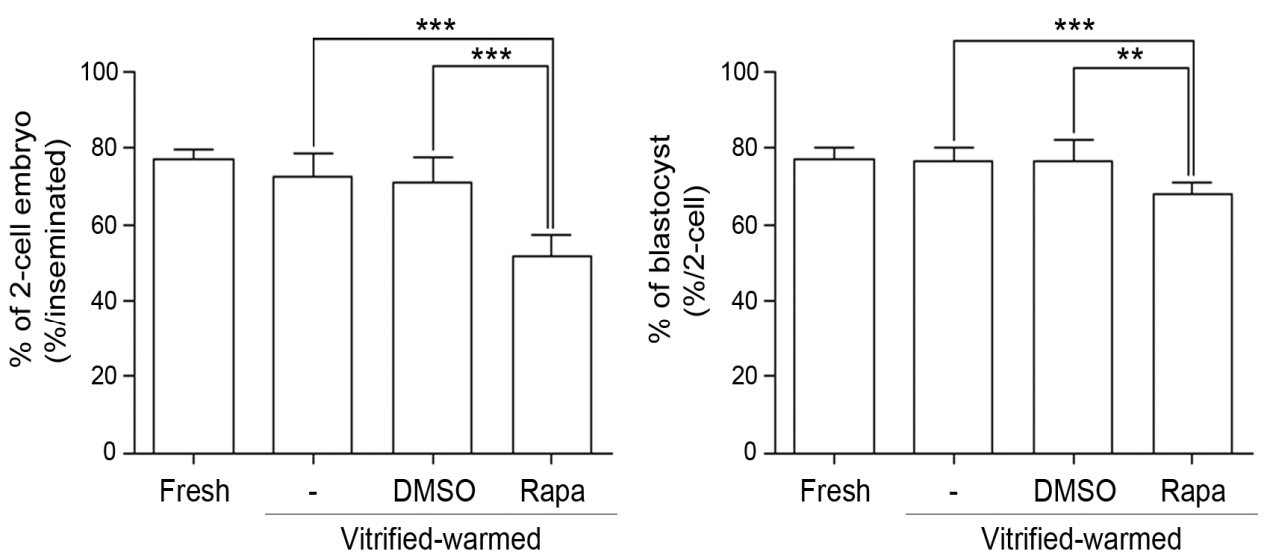

\begin{tabular}{ccccccc}
\hline \multirow{2}{*}{ Group } & \multicolumn{5}{c}{ Number of } \\
\cline { 2 - 7 } & $\begin{array}{c}\text { Vitrified } \\
\text { oocytes }\end{array}$ & $\begin{array}{c}\text { Survived } \\
\text { oocytes (\%) }\end{array}$ & $\begin{array}{c}\text { Inseminated } \\
\text { oocytes }\end{array}$ & $\begin{array}{c}\text { Two-cell embryo } \\
(\% \text { /inseminated) }\end{array}$ & $\begin{array}{c}\text { Blastocysts } \\
\text { (\%/two-cell) }\end{array}$ \\
\hline \multirow{2}{*}{ Fresh } & - & - & 228 & $176(77.2)$ & $135(76.7)$ \\
\hline \multirow{2}{*}{$\begin{array}{c}\text { Vitrified } \\
\text {-warmed }\end{array}$} & DMSO & 218 & $190(87.2)$ & 190 & $134(70.5)$ & $102(76.1)$ \\
\cline { 2 - 7 } & 100 nM Rapa & 217 & $206(94.9)$ & 206 & $106(51.5)$ & $72(67.9)$ \\
\hline
\end{tabular}

Figure 6. Effect of autophagy induction on survival, fertilization, and development in oocytes treated with rapamycin during warming and recovery after vitrification. Graphs show the percentage of 2-cell embryos and blastocysts after in vitro fertilization. The table shows the total number and percentage of oocytes that were used in the barograms. Data represent the mean of six independent experiments $(\mathrm{n}=$ 6 ), while error bars represent the mean \pm standard deviation. Statistical significance was analyzed by Student's $t$ test (one-tailed). ** $\mathrm{p}<0.01, * * * \mathrm{p}<0.001$.

after vitrification has a negative effect on fertilization and development. We propose that the extent of autophagic activation in MII oocytes could further be developed as a marker to predict developmental competence after cryopreservation. Cryopreserved MII oocytes with little or no autophagic activation may thus predict better embryo quality.

\section{CONFLICT OF INTEREST}

We certify that there is no conflict of interest with any financial organization regarding the material discussed in the manuscript.

\section{ACKNOWLEDGMENTS}

This study was supported by a grant of the Korea Health Technology R\&D Project, Ministry of Health \& Welfare, Republic of Korea (HI12C0055).

\section{REFERENCES}

Adastra, K. L., M. M. Chi, J. K. Riley, and K. H. Moley. 2011. A differential autophagic response to hyperglycemia in the developing murine embryo. Reproduction 141:607-615.

Bang, S., H. Shin, H. Song, C. S. Suh, and H. J. Lim. 2014.
Autophagic activation in vitrified-warmed mouse oocytes. Reproduction 148:11-19.

Boya, P., F. Reggiori, and P. Codogno. 2013. Emerging regulation and functions of autophagy. Nat. Cell. Biol. 15:713-720.

Castedo, M., K. F. Ferri, and G. Kroemer. 2002. Mammalian target of rapamycin (mTOR): pro- and anti-apoptotic. Cell. Death. Differ. 9:99-100.

Cha, S. K., B. Y. Kim, M. K. Kim, Y. S. Kim, W. S. Lee, T. K. Yoon, and D. R. Lee. 2011. Effects of various combinations of cryoprotectants and cooling speed on the survival and further development of mouse oocytes after vitrification. Clin. Exp. Reprod. Med. 38:24-30.

Chiang, G. G. and R. T. Abraham. 2005. Phosphorylation of mammalian target of rapamycin (mTOR) at Ser-2448 is mediated by p70S6 kinase. J. Biol. Chem. 280:25485-25490.

Choi, S., H. Shin, H. Song, and H. J. Lim. 2014. Suppression of autophagic activation in the mouse uterus by estrogen and progesterone. J. Endocrinol. 221:39-50.

Gualtieri, R., V. Mollo, V. Barbato, I. Fiorentino, M. Iaccarino, and R. Talevi. 2011. Ultrastructure and intracellular calcium response during activation in vitrified and slow-frozen human oocytes. Hum. Reprod. 26:2452-2460.

Heitman, J., N. R. Movva, and M. N. Hall. 1991. Targets for cell cycle arrest by the immunosuppressant rapamycin in yeast. Science 253:905-909.

Jo, J. W., B. C. Jee, J. R. Lee, and C. S. Suh. 2011. Effect of antifreeze protein supplementation in vitrification medium on mouse oocyte developmental competence. Fertil. Steril. 
96:1239-1245.

Kapahi, P., D. Chen, A. N. Rogers, S. D. Katewa, P. W. Li, and E. L. Thomas, and L. Kockel. 2010. With TOR, less is more: A key role for the conserved nutrient-sensing TOR pathway in aging. Cell. Metab. 11:453-465.

Klionsky, D. J., F. C. Abdalla, H. Abeliovich, R. T. Abraham, A. Acevedo-Arozena, and K. Adeli, et al. 2012. Guidelines for the use and interpretation of assays for monitoring autophagy. Autophagy 8:445-544.

Kogasaka, Y., Y. Hoshino, Y. Hiradate, K. Tanemura, and E. Sato. 2013. Distribution and association of mTOR with its cofactors, raptor and rictor, in cumulus cells and oocytes during meiotic maturation in mice. Mol. Reprod. Dev. 80:334-48.

Lee, J. E., H. A. Oh, H. Song, J. H. Jun, C. R. Roh, H. Xie, S. K. Dey, and H. J. Lim. 2011a. Autophagy regulates embryonic survival during delayed implantation. Endocrinology 152:2067-2075.

Lee, S. E., K. C. Hwang, S. C. Sun, Y. N. Xu, and N. H. Kim. 2011b. Modulation of autophagy influences development and apoptosis in mouse embryos developing in vitro. Mol. Reprod. Dev. 78:498-509.

Lee, S. E., S. C. Sun, H. Y. Choi, S. J. Uhm, and N. H. Kim. 2012. mTOR is required for asymmetric division through small GTPases in mouse oocytes. Mol. Reprod. Dev. 79:356-366.

Lim, H. J. and H. Song. 2014. Evolving tales of autophagy in early reproductive events. Int. J. Dev. Biol. 58:183-187.

Martínez-Burgos, M., L. Herrero, D. Megías, R. Salvanes, M. C. Montoya, A. C. Cobo, and Juan A. Garcia-Velasco. 2011. Vitrification versus slow freezing of oocytes: Effects on morphologic appearance, meiotic spindle configuration, and DNA damage. Fertil. Steril. 95:374-377.

Mayer, S., C. Wrenzycki, and W. Tomek. 2014. Inactivation of mTor arrests bovine oocytes in the metaphase-I stage, despite reversible inhibition of 4E-BP1 phosphorylation. Mol. Reprod. Dev. 81:363-375.

Mizushima, N., B. Levine, A. M. Cuervo, and D. J. Klionsky. 2008. Autophagy fights disease through cellular self-digestion. Nature 451:1069-1075.

Mizushima, N., A. Yamamoto, M. Matsui, T. Yoshimori, and Y. Ohsumi. 2004. In vivo analysis of autophagy in response to nutrient starvation using transgenic mice expressing a fluorescent autophagosome marker. Mol. Biol. Cell. 15:11011111.
Neufeld, T. P. 2010. TOR-dependent control of autophagy: Biting the hand that feeds. Curr. Opin. Cell. Biol. 22:157-168.

Sancak, Y., T. R. Peterson, Y. D. Shaul, R. A. Lindquist, C. C. Thoreen, L. Bar-Peled, and David M. Sabatini. 2008. The Rag GTPases bind raptor and mediate amino acid signaling to mTORC1. Science 320:1496-1501.

Saragusty, J. and A. Arav. 2011. Current progress in oocyte and embryo cryopreservation by slow freezing and vitrification. Reproduction 141:1-19.

Singh, R. and A. M. Cuervo. 2011. Autophagy in the cellular energetic balance. Cell. Metab. 13:495-504.

Song, B. S., J. S. Kim, Y. H. Kim, B. W. Sim, S. B. Yoon, and J. J. Cha, S.-A Choi, H.-J. Yang, S.-E. Mun, Y.-H. Park, K.-J. Jeong, J.-W. Huh, S.-R. Lee, S.-H. Kim, S.-U. Kim, and K.-T. Chang. 2014. Induction of autophagy during in vitro maturation improves the nuclear and cytoplasmic maturation of porcine oocytes. Reprod. Fertil. Dev. 26:974-981.

Susor, A., D. Jansova, R. Cerna, A. Danylevska, M. Anger, T. Toralova, R. Malik, J. Supolikova, M. S. Cook, J. S. Oh, and M. Kubelka. 2015. Temporal and spatial regulation of translation in the mammalian oocyte via the mTOR-eIF4F pathway. Nat. Commun. 6:6078.

Tsukamoto, S., T. Hara, A. Yamamoto, S. Kito, N. Minami, T. Kubota, K. Sato, and T. Kokubo. 2014. Fluorescence-based visualization of autophagic activity predicts mouse embryo viability. Sci. Rep. 4:4533.

Tsukamoto, S., A. Kuma, M. Murakami, C. Kishi, A. Yamamoto, and N. Mizushima. 2008. Autophagy is essential for preimplantation development of mouse embryos. Science 321:117-20.

Yamamoto, A., N. Mizushima, and S. Tsukamoto. 2014. Fertilization-induced autophagy in mouse embryos is independent of mTORC1. Biol. Reprod. 91:7.

Yang, Z. and D. J. Klionsky. 2010. Eaten alive: A history of macroautophagy. Nat. Cell. Biol. 12:814-822.

Yu, L., C. K. McPhee, L. Zheng, G. A. Mardones, Y. Rong, J. Peng, N. Mi, Y. Zhao, Z. Liu, F. Wan, D. W. Hailey, V. Oorschot, J. Klumperman, E. H. Baehrecke, and M. J. Lenardo. 2010. Termination of autophagy and reformation of lysosomes regulated by mTOR. Nature 465:942-946. 